\title{
Rhythmical Discharges Recorded from Cervical and Trunk Muscle Nerves Produced by Stimulation of Mesencephalic Locomotor Region (MLR) in the Decerebrated Cat
}

\author{
Kuniaki TAKAHASHI, Hiroko NISHIMURA, Naomi WADA, and Mikihiko TOKURIKI \\ Department of Veterinary Physiology, Faculty of Agriculture, Yamaguchi University, Yamaguchi 753, Japan \\ (Received 15 April 1997/Accepted 10 June 1997)
}

ABSTRACT. This experiment investigated the effects of mesencephalic locomotor region (MLR) stimulation on cervical and trunk motoneurons in decerebrated cats. The experiments were performed on 28 adult cats $(2.1-3.9 \mathrm{~kg})$ of either sex. Stimulation of MLR produced the rhythmical discharge of muscle nerves of the splenius (SP), the longissimus lumborum (LL) and the obliquus externus abdominis (OEA). There was a close relationship between rhythmical discharge of SP, LL or OEA muscle nerve and hindlimb muscle nerve. This fact suggests that rhythmical discharges of cervical and trunk muscle nerves relate to locomotion. - KEY wORDs: cervical and trunk muscle nerves, feline, MLR.

J. Vet. Med.Sci. 59(10): 951-952, 1997

In the precollicular decerebrated cat, electrical stimulation of the midbrain produces the stepping of all four limbs on a moving belt $[6,7]$ and rhythmic discharge of nerves in the muscle of all four limbs in immobilized cats $[1,3,5]$. The effective area in the midbrain corresponds to the caudal part of the cuneiform nucleus [4], and has been called the mesencephalic locomotor region (MLR) [8]. However, the effects of MLR stimulation have not previously been reported on cervical and trunk muscle motoneurons. In the present experiments, the effects of MLR stimulation on motoneurons innervating the cervical and trunk muscles were studied.

The experiments were performed on 28 adult cats (2.1$3.9 \mathrm{~kg}$ ) of either sex. Cats were anesthetized with a mixture of halothane and nitrous oxide. The left and right carotid arteries were cannulated to monitor blood pressure and allow perfusion with fixative solution, respectively. The left muscle nerves innervating the splenius (SP:C3), the longissimus lumborum (LL: L2), the obliquus externus abdominis (OEA: L2), the posterior biceps and semitendinosus (PBST) and the lateral gastrocnemius and soleus (LGS), were carefully isolated from surrounding tissue. Animals were decerebrated by passing a spatula rostroventrally from a line about $1 \mathrm{~mm}$ rostral to the superior colliculus and aspirating tissue rostral to the transection under anesthesia with a mixture of halothane and nitrous oxide. After decerebration, anesthesia was discontinued. The cat was immobilized with pancronium bromide $(0.4$ $\mathrm{mg} / \mathrm{h}$ i.v.) and artificially ventilated. End-tidal $\mathrm{CO}_{2}$ concentration was monitored and maintained at approximately $4 \%$ by adjusting the respiratory rate or tidal volume. The rectal temperature was monitored and maintained at close to $38^{\circ} \mathrm{C}$ with a heating mat. Discharges from muscle nerves were recorded with bipolar silver electrodes. The left MLR was stimulated using tungsten monopolar electrode inserted into the midbrain (around P2, L4 and H0 in Horsley-Clarke coordinates; $20 \mathrm{~Hz}$ in frequency, $0.1 \mathrm{msec}$ in duration, $100 \mu \mathrm{A}$ or less in current) $[2,7]$. Discharges from muscle nerves were amplified using a differential amplifier (NEC, Sanei, bioelectrical amplifier1253 ) and recordings were stored on magnetic tape (band width: d.c.-2.5 kHz, TEAC, RD-135T). At the end of the experiment, stimulation sites in the midbrain were determined histologically.

Stimulation of MLR produced the rhythmical discharges in SP, LL, OEA, PBST and LGS muscle motoneurons. Rhythmical discharges in SP, LL, OEA, PBST and LGS muscle nerves were observed in $4(14 \%), 7(25 \%), 4(14 \%)$, $14(50 \%)$ and $14(50 \%)$ of 28 cats, respectively. The rates of rhythmical discharges appearing in neck and trunk muscles nerves was lower than that appearing in hindlimb muscles nerves. The appearance rates of rhythmical discharges were not influenced by increasing intensity of MLR stimulation in all muscles nerves. Furthermore, the kinds of muscles nerves which showed rhythmical discharge were not independent with stimulating sites of MLR.

Figure 1 shows an example of rhythmical discharge recorded from SP, PBST and LGS muscle nerves. The line below the recording indicates the duration of MLR stimulation. During MLR stimulation, a stable discharge pattern developed in SP, PBST and LGS muscle nerves and then disappeared at the end of MLR stimulation, indicating that rhythmical discharges of SP, PBST and LGS were produced by MLR stimulation. The rhythmical discharge of SP was synchronized with that of PBST and an alternating pattern of discharge between SP and LGS was observed.

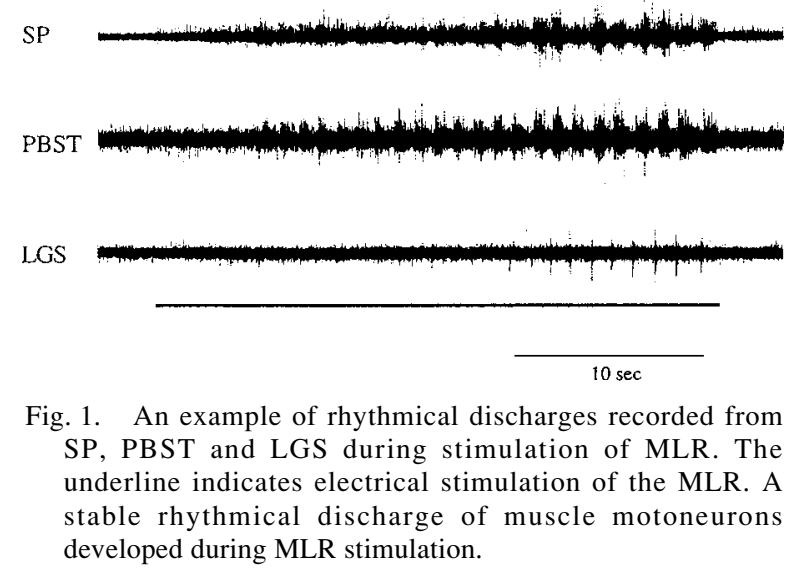


A

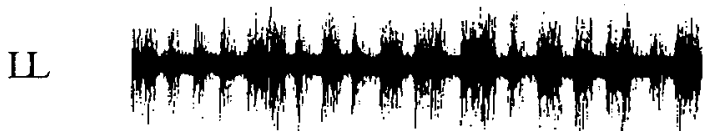

PBST

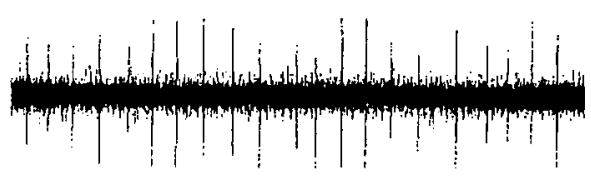

LGS

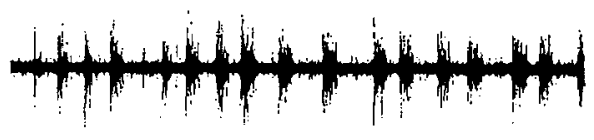

B

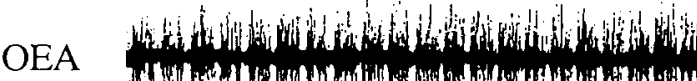

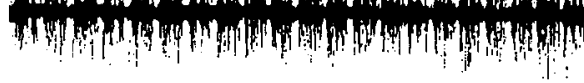

PBST

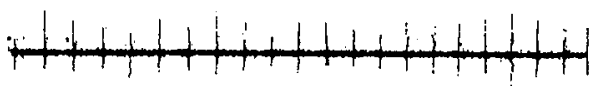

LGS

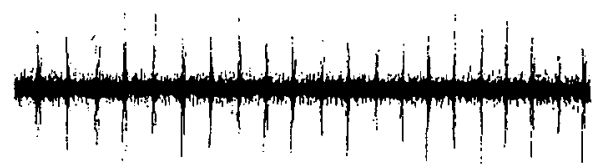

Fig. 2. Examples of rhythmical discharges recorded from LL (A) and OEA (B) muscle nerves. The rhythmical discharges recorded from LL and OEA muscle nerves showed a close relationship with those of PBST and LGS.

Figure 2 shows examples of rhythmical discharges recorded from trunk muscle nerves during MLR stimulation. MLR stimulation produced rhythmical discharge of LL (Fig. 2A) and OEA (Fig. 2B) muscle nerves. The discharge of LL and OEA muscle nerves showed a close relationship with discharge of the hindlimb muscle nerves.

The results of the present experiments clearly showed that rhythmical discharge of the cervical muscle (SP) and trunk muscle motoneurons (LL and OEA) similar to those in hindlimbs muscle motoneurons were produced by MLR stimulation. Furthermore, discharges recorded from the cervical and trunk muscle nerves showed a close relationship with hindlimb muscle nerve discharges. This fact suggests that rhythmical discharges of cervical and trunk muscle nerves produced by MLR stimulation relate to locomotion.

\section{REFERENCES}

1. Anemiya, M. and Yamaguchi, T. 1984. Neurosci. Lett. 50: 91-96.

2. Berman, A. L. 1968. In: The Brain Stem of the Cat. University of Wisconsin Press, Madison.

3. Feldman, A. G. and Orlovsky, G. N. 1975. Brain Res. 84: 181-194.

4. Garcia-Rill, E. and Skinner, R. D. 1986. Macmillan Press (Lond.) 77-103.

5. Jordan, L. M., Pratt, C. A., and Menzies, J. E. 1979. Brain Res. 177: 204-207.

6. Shik, M. L., Severin, F. V., and Orlovsky, G. N. 1966. Biofizika 11: 659-666.

7. Shik, M. L., Orlovsky, G. N., and Severin, F. V. 1966. Biofizika 11: 879-886.

8. Shik, M. L. and Orlovsky, G. N. 1976. Physiol. Rev. 56: 465501. 